Scientific Visualization, 2020, volume 12, number 4, pages 1 - 8, DOI: 10.26583/sv.12.4.01

\title{
Verification of Cause-and-Effect Relationships in Cognitive Models Using Visualization Metaphors of Fuzzy Cognitive Maps
}

\author{
R.A. Isaev ${ }^{1}$, A.G. Podvesovskii ${ }^{2}$ \\ Bryansk State Technical University \\ 1 ORCID: 0ooo-0003-3263-4051, ruslan-isaev-32@yandex.ru \\ 2 ORCID: 00oo-0002-1118-3266, apodv@tu-bryansk.ru
}

\begin{abstract}
The paper presents the continuation of the authors' research in the field of visualization of cognitive models based on fuzzy cognitive maps. Application of visualization metaphors of fuzzy cognitive maps for verification of cause-and-effect relationships in cognitive models is considered. It is shown that increasing the effectiveness of cognitive model verification is possible by activating analyst's cognitive potential. The most natural way of such activation is to increase cognitive clarity of the model through the use of visualization capabilities. For this purpose, a number of metaphors for visualizing fuzzy cognitive maps have been proposed, aimed at increasing their cognitive clarity during verification. Each of the metaphors is focused on the visualization of a certain type of fragments of a fuzzy cognitive map potentially containing errors, redundancy or incompleteness and therefore of interest from the point of view of verification. Examples are given of applying the proposed visualization metaphors.
\end{abstract}

Keywords: fuzzy cognitive map, graph visualization, cognitive clarity, visualization metaphor, cognitive model verification.

\section{Introduction}

This paper continues a series of publications of the authors' research materials in the field of visualization of cognitive models based on fuzzy cognitive maps (FCM). An FCM reflects a researcher's subjective idea of a system in the form of a set of semantic categories (called factors or concepts) and a set of causal relationships between them [1]. Thus, an FCM can be visualized in the form of a weighted directed graph the vertices of which correspond to concepts and edges - to cause-and-effect relationships.

One of the conditions for effective work with a cognitive model is to ensure its visual representation. In [2, 3], the authors proposed an approach to FCM visualization based on the visualization metaphor concept. Visualization metaphor traditionally includes two components: spatial metaphor and representation metaphor [4].

The spatial metaphor defines general principles of transferring a visualized object into the visual model space. With regard to an FCM, such a metaphor is based on graph visualization algorithms and formalized criteria of cognitive clarity [3]. These criteria describe requirements for the FCM visual image quality. Observing these requirements simplifies visual perception of the cognitive model by the analyst. This leads to a general increase in the speed of work with the model and also helps to reduce the number of errors made at various stages of modeling.

The representation metaphor used below is responsible for finalizing the resulting visual image in order to identify its components that are most important in the context of the problem being solved. A number of different representation metaphors are used in the visualization of the FCM taking into account the analyst's needs at different stages of cognitive modeling. 
The paper investigates capabilities of FCM visualization when solving one of the important tasks of cognitive modeling - namely, cognitive model verification. The research is based on the hypothesis of efficiency increase in verification of cause-and-effect relationships within a cognitive model by increasing cognitive clarity of the visual image of the corresponding FCM.

\section{Cognitive model verification task}

Cognitive model verification is one of the most important stages in their construction since reliability of the results of subsequent modeling largely depends on the successful implementation of verification. The task of verifying a cognitive model is aimed at identifying its possible inconsistency with the modeled system itself. Such inconsistency can be expressed in the following basic forms.

The cognitive model may lack concepts that reflect important parameters of the modeled system, or, conversely, there may be redundant concepts that are not important in relation to the modeling goal.

A set of cause-and-effect relationships given on a set of concepts can be characterized by both incompleteness and redundancy.

Errors can be made when setting parameters of cause-and-effect relationships (direction, sign, intensity).

Search techniques and eliminating inconsistencies of the first type require highly qualified experts and deep understanding of the subject area and, as a rule, are the most difficult to formalize. One of the possible approaches here may be ontological engineering [5].

Errors made in the parametric identification of the model (the third type of discrepancy) are the least obvious for detection and most often can be detected directly from the results of modeling, based on the analysis of their plausibility [6]. At the same time, certain reliability control of parameters of FCM relationships can be performed within the framework of the identification methods themselves [7].

The proposed research focuses on the second type of discrepancy as the easiest one for applying formal verification methods. Besides, as will be shown below, in this case, it becomes possible to effectively combine formal methods with methods based on the activation of the analyst's cognitive capabilities [8].

\section{General methodology for verification of cause-and- effect relationships in cognitive models}

Let us describe the proposed cognitive model verification methodology, focused on identifying and eliminating errors on a set of cause-and-effect relationships and based on the use of graph search algorithms and FCM visualization metaphors. Fig. 1 shows a generalized algorithm that implements this technique.

At the first stage, a search is carried out within the FCM for all structural elements that are of interest from the point of view of verification of cause-and-effect relationships in a cognitive model. Note that the methodology is invariant with respect to the specific types of structural elements taken into account. In this paper, three types of structural elements are considered in detail.

When identifying the redundancy of a set of relationships, the most important for analysis are such types of elements of the cognitive graph structure as directed cycles and pairs of transitive paths. The importance of directed cycles stems from the fact that they, representing feedback loops, in some cases can lead to a violation of cognitive model stability in the course of its scenario analysis. Pairs of transitive paths are indicative of the existence of alternative mechanisms for transferring influence between concepts. Such mechanisms must be assessed by the analyst, on the one hand, for their mutual consistency, and on the other hand, for the appropriateness of their simultaneous reflection in the model. 


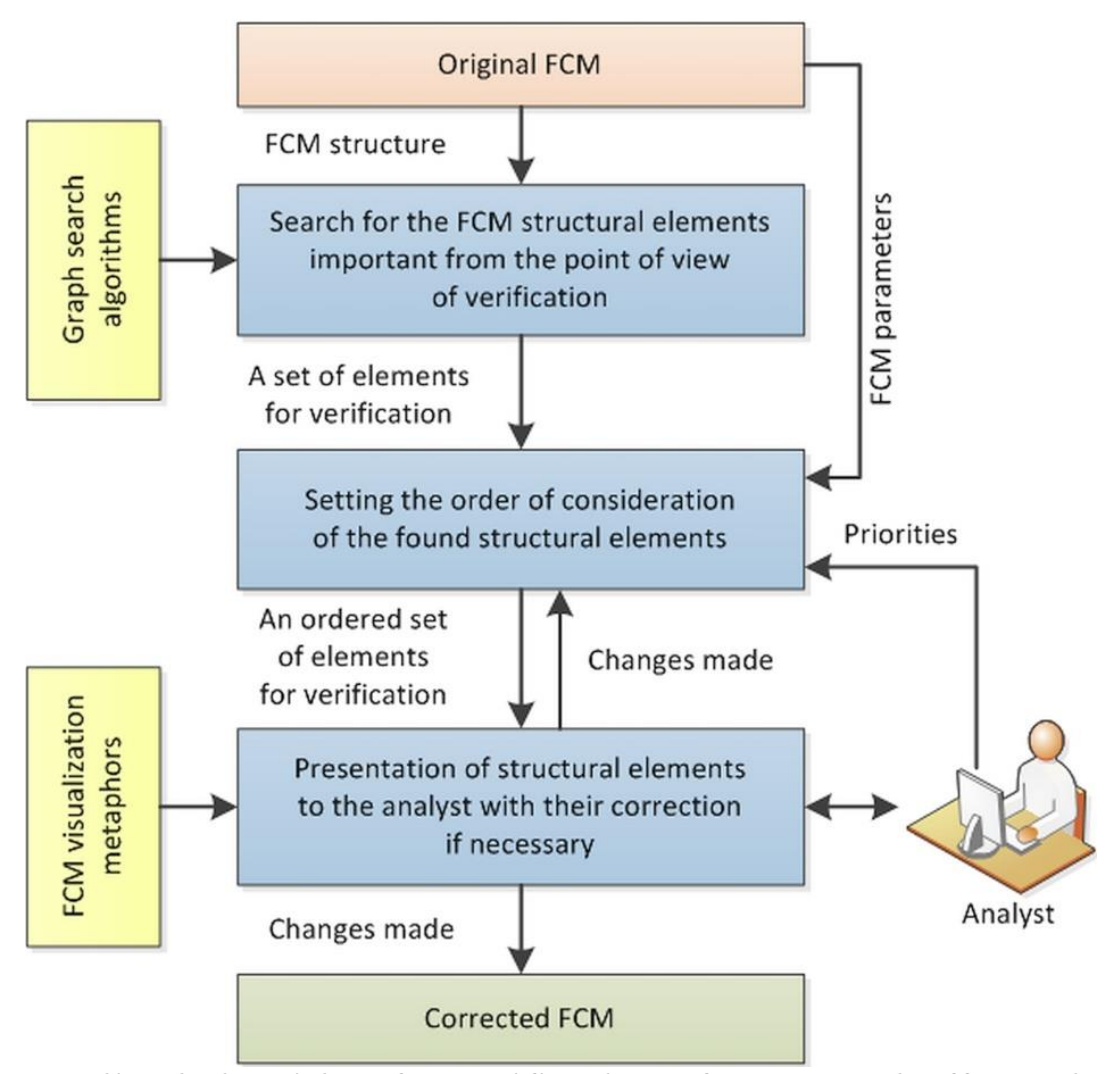

Fig. 1. Generalized algorithm for verification of cause-and-effect relationships in a cognitive model using FCM visualization metaphors

The problem of search for directed cycles and pairs of transitive paths in an FCM belongs to common problems in graph theory. Among a number of possible ways to solve it, the method described below is of the greatest practical interest.

1. A search is performed for all cycles in an undirected graph which can be associated with the FCM under study (by eliminating the orientation of the edges). This search can be performed based on the depth first search algorithm [9].

2. For each of the found undirected cycles, it is necessary to establish whether it corresponds to a directed cycle or a pair of transitive paths in the original FCM. For this purpose, an algorithm can be used consisting of the following steps.

2.1. Selection in the found cycle of any vertex from which there is at least one outgoing edge.

2.2. Transition to the next vertex along this outgoing edge.

2.3. Traversing the cycle in the originally selected direction. As this takes place, each direction-relative change of the next edge is recorded, and the number of such cases is counted.

2.4. The traversal ends with the return to the original vertex. If during the traversal not a single case of a change in the direction of the edges was recorded, then a directed cycle has been detected. If one or two cases were recorded, then a pair of transitive paths has been found.

As mentioned earlier, many relationships between concepts can be characterized not only by redundancy, but also by incompleteness, which is understood as the lack of necessary relationships. It should be noted here that absence of directed paths between some pairs of concepts in a cognitive graph is a typical situation when building a cognitive model. As a consequence, even in the long term, changes in the states of some concepts will not affect the states of a number of other concepts. From the point of view of object interpretation, this means cause-and-effect independence of the corresponding parameters of the modeled system from each other; this is quite admissible. Nevertheless, in a number of cases, a missing relationship occurs by mistake during the FCM construction, and such situations require detection and correction.

Pairs of concepts which lack directed paths between them can be easily identified based on the operation of transitive closure of a cognitive matrix corresponding to the FCM under 
study. A sign of the absence of a path between the concepts is the equality to zero of the corresponding element of the transitively closed matrix.

Thus, as a result of performing the first stage of the generalized algorithm, a set of FCM structural elements of three types is formed: directed cycles, pairs of transitive paths and missing paths between concepts. Further, these elements must be reviewed by the analyst for the necessity to correct them. In this case, almost always, due to the large volume of the formed set as well as from considerations of effective use of the available time, it becomes necessary to specify the order of presentation of its elements to the analyst. This corresponds to the second stage of the generalized algorithm.

When determining this order, in addition to subjective preferences of the analyst himself, it is necessary to take into account objective factors that characterize the significance of a particular structural element in the context of verification of this cognitive model.

So, in case of directed cycles, it is advisable to take into account that the risk of FCM stability disruption is primarily due to the presence of cycles with a positive weight (the weight of a cycle refers to the product of the weights of influences included in it): concepts in such a cycle tend to intensify their own state changes. Therefore, if there are several such cycles, priority should be given to the cycles with the highest weight.

By analogy with cycles, any directed path in the graph can also be assigned a weight, defined in a similar way. A path with a positive weight corresponds to the mechanism of strengthening one concept by another, and with a negative weight - to a mechanism of weakening. Thus, a pair of transitive paths can describe both contradictory and mutually confirming (and therefore, probably redundant) chains of influences of one concept on another. Obviously, in the context of verification, the most significant are the pairs that include paths with the highest absolute weight.

When analyzing the found pairs of unrelated concepts, it is advisable to give the highest priority to such pairs adding a relationship between which will have the most significant impact on the modeling results. This question will be explored in more detail providing a specific example in the next section.

Using the assigned order, at the final third stage of the generalized algorithm, the found structural elements of the FCM are presented to the analyst. Moreover, for each type of structural element its own FCM visualization metaphor is used. Let us take a closer look at these metaphors.

\section{Visualization metaphors of fuzzy cognitive maps used in the process of verifying cause-and-effect relationships in cognitive models}

Let us consider some FCM visualization metaphors that can be used in the process of verifying cause-and-effect relationships in cognitive models. The use of these and other similar metaphors increases cognitive clarity of verified models, which will help to activate the analyst's cognitive abilities when solving a number of specific tasks during verification. To illustrate results of applying the proposed metaphors, we will use the FCM shown in Fig. 2. 


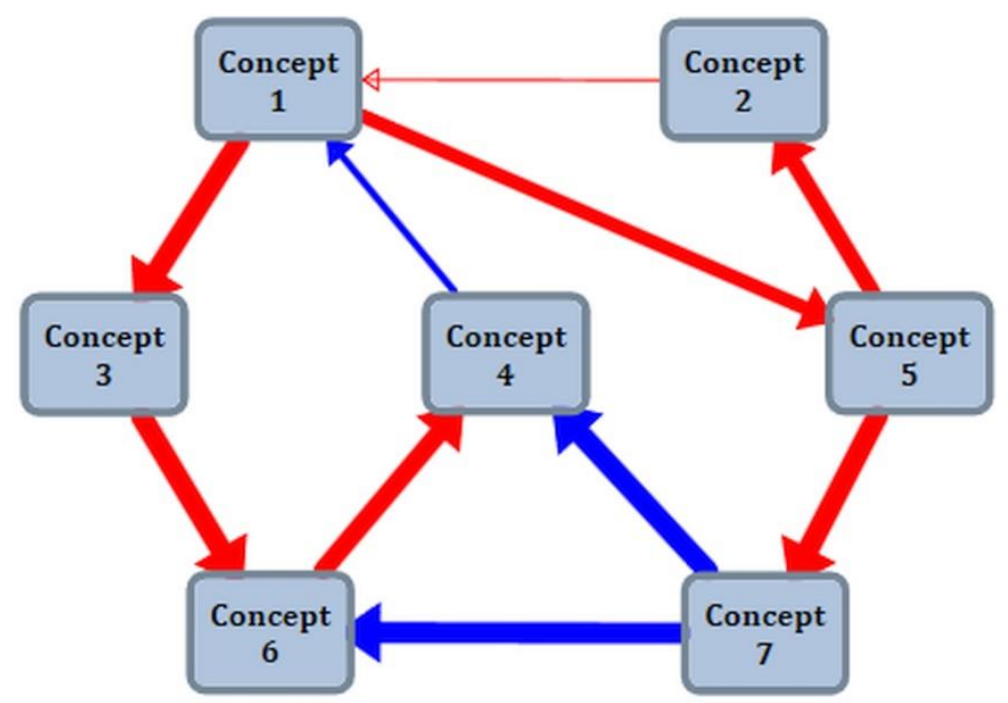

Fig. 2. Example of an FCM subject to verification

\subsection{Visualization of oriented cycles}

Let us consider a visualization metaphor designed to display directed cycles found within an FCM. Suppose one of the cycles is set for the visualization. The essence of the spatial component of this metaphor consists in depicting the FCM in such a way that the criterion of unidirectionality of successive edges is maximized on the set of edges included in a given cycle. Less formally, the metaphor seeks to place the vertices of the cognitive graph in such a way as to provide a unidirectional image of as many edges within the cycle as possible. It has been noted [Ошибка! Источник ссылки не найден.о] that such placement contributes to the cycle coverage "at a glance". In this case, it is advisable to choose the direction "left-toright" or "top-down" as the priority direction (that is, the direction of most edges). This is due to the criterion of optimizing edge directions which is also taken into account [3].

The corresponding representation metaphor is characterized by the concentration of the analyst's attention directly on the cycle under consideration. A simple solution to this problem could be complete absence of images of "excess" sections of the FCM. However, this approach has an obvious disadvantage of removing the context useful for verification from the analyst's perception. Therefore, it seems more rational to depict all the FCM elements that are not included in the cycle semi-transparent. It should also be noted that it is advisable to individually adjust the degree of transparency taking into account peculiarities of a particular analyst's perception.

Fig. 3 shows an example of application of this metaphor to the test FCM when visualizing the cycle "1-5-7-6-4-1". It is easy to see that restructuring the FCM image is much better (compared to the original metaphor) in attracting the analyst's attention to the selected cycle. This allows us to speak of an increase in cognitive clarity of the model in the context of the problem under consideration.

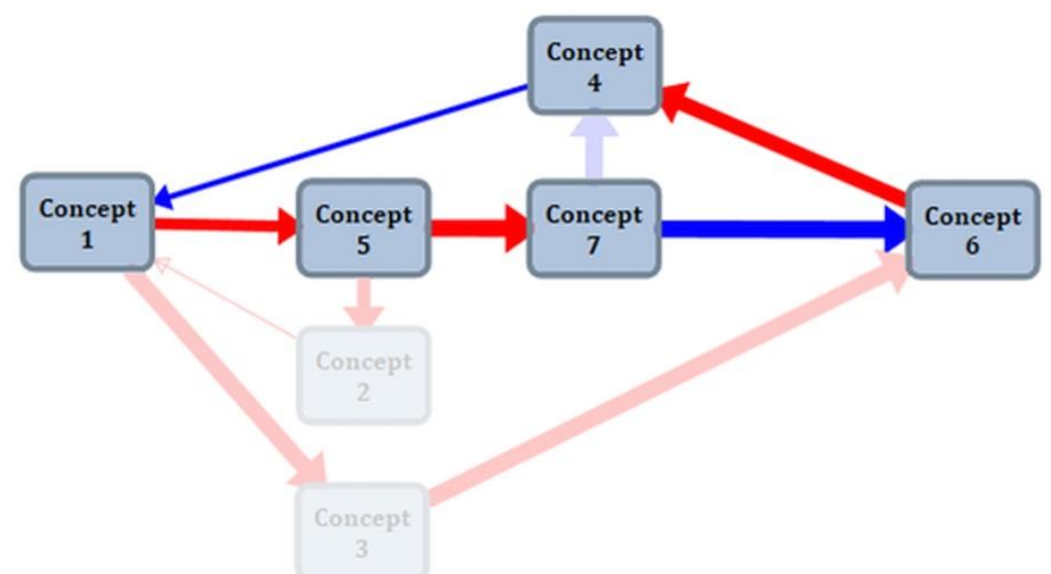

Fig. 3. Example of applying the cycle visualization metaphor 


\subsection{Visualization of pairs of transitive paths}

The next visualization metaphor is intended to depict pairs of transitive paths between concepts. As in the previous case, the spatial component of this metaphor takes into account the criterion of unidirectionality of successive edges but additionally maximizes the symmetry of the subgraph subject to visualization [3]. By analogy with the previous case, the representation metaphor uses the effect of a semi-transparent image of "excess" graph sections to focus the analyst's attention on the selected transitive paths.

Fig. 4 shows an example of this metaphor application when visualizing a pair of paths "1-3-64" and "1-5-7-4". Due to equal path lengths, it was possible to ensure the symmetry of the target subgraph about the horizontal axis.

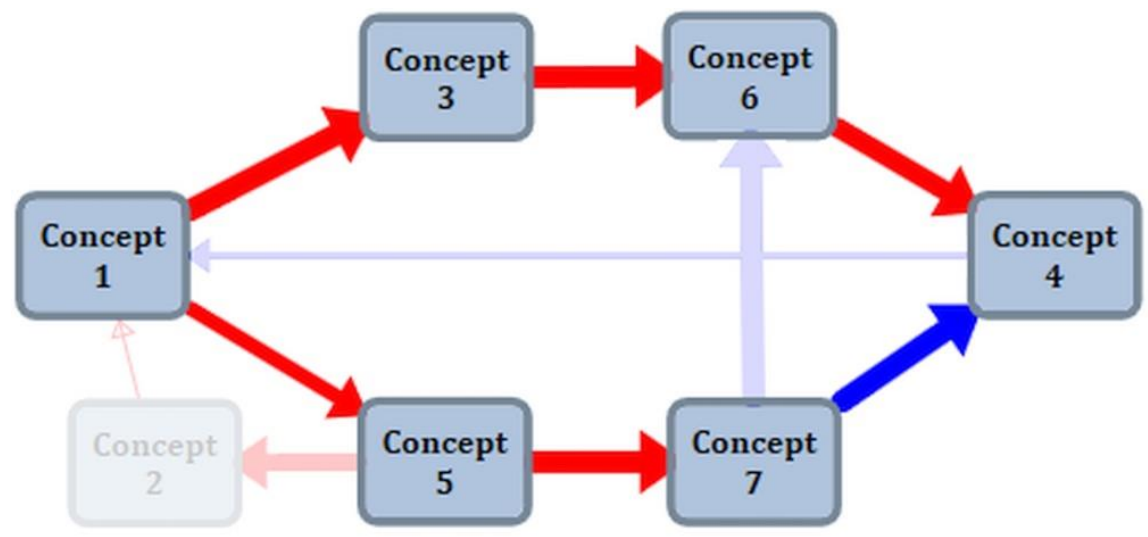

Fig. 4. Example of metaphor application for visualizing pairs of transitive paths

By analogy with the previous metaphor, the priority direction of the edges can be either "leftto-right" or "top-down", depending on the analyst's preferences.

\subsection{Visualization of a missing relationship between concepts}

Let the FCM in Fig. 2 initially lack relationship directed from Concept 6 to Concept 4 . It is easy to see that this leads to the absence of oriented paths from Concept 3 to all concepts except Concept 6 , as well as from Concept 6 to all concepts.

Let us suppose that a relationship is added from Concept 6 to any of the concepts numbered $1,2,4,5$. Obviously, this will lead to the emergence of oriented paths from Concept 6 itself to all concepts, as well as from Concept 3 to all concepts. If such a relationship is added from Concept 3, then Concept 6 will remain isolated. Therefore, it is advisable to assign a higher priority to considering Concept 6 as a concept-cause.

Further, it is required to determine the order of presentation of potential conceptconsequences, that is, concepts with numbers 1, 2, 4, 5. A possible solution here may be to focus on the intensity of influences exerted by these concepts on the other FCM concepts. This information can also be obtained from a transitively closed matrix. The greatest total influence on the concepts within the FCM is exerted by Concept 1.

An example of using a visualization metaphor taking into account the above reasoning is shown in Fig. 5. The analyst is invited to add a relationship from Concept 6 to Concept 1, and he can either agree with this proposal or refuse it. If the analyst agrees to add a relationship, then he needs to set its parameters, which, in turn, requires the use of methods of FCM parametric identification [7].

An important feature of the proposed visualization metaphor is the possibility of adjusting its spatial component in order to increase cognitive clarity of the visual image of the FCM. So, if the concepts presented to the analyst in order to add a relationship are situated far from each other and are separated by other elements of the FCM, then visual image rebuilding is performed, aimed simultaneously at the spatial convergence of these concepts and at maintaining the usual location of the remaining FCM elements. Fig. 6 exemplifies how the metaphor works in such a situation. It should be noted that from the point of view of automating visual 
image correction, an approach based on the simulated annealing method proposed in [11] is of interest.

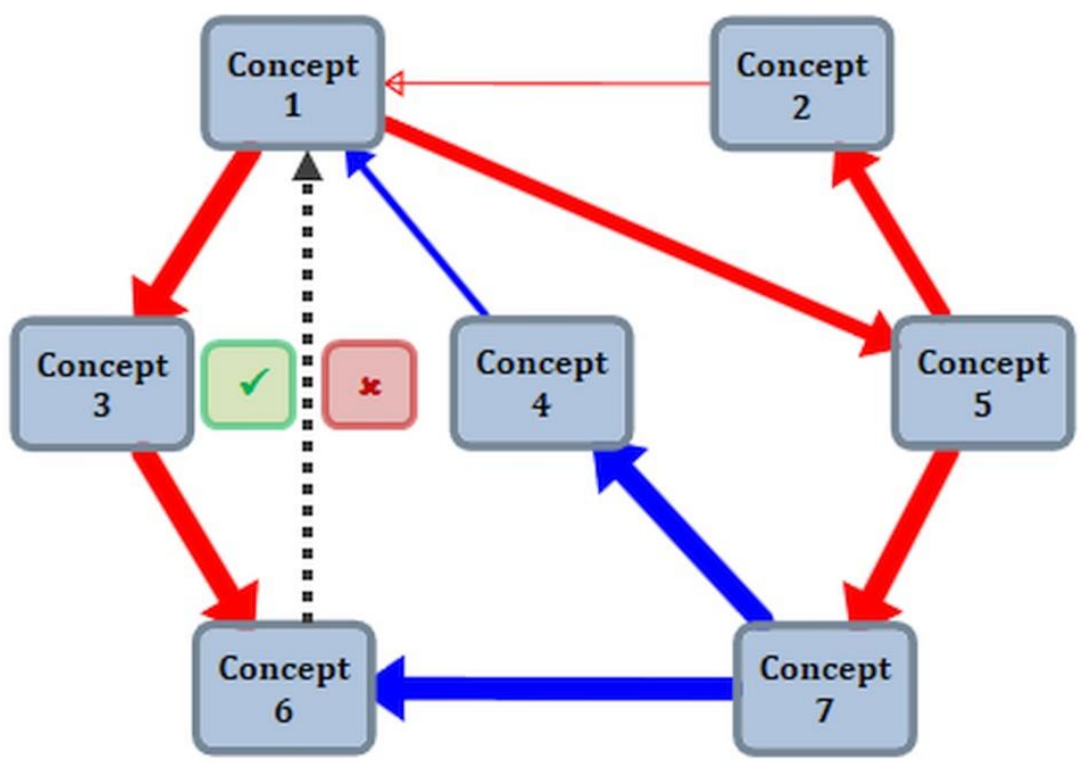

Fig. 5. Example of using a visualization metaphor to eliminate a missing relationship between concepts (the case of preserving the original spatial metaphor)

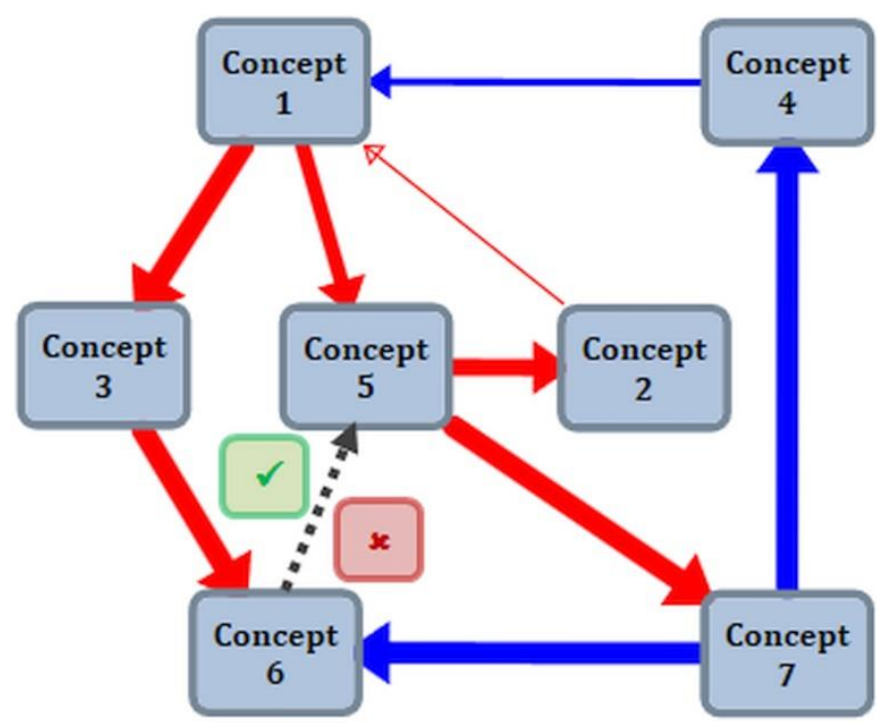

Fig. 6. Example of using a visualization metaphor to eliminate a missing relationship between concepts (the case of spatial metaphor correction)

\section{Conclusion}

The paper presents possibilities of applying the approach to FCM visualization based on visualization metaphors for verifying cause-and-effect relationships in fuzzy cognitive models.

A methodology for verifying cause-and-effect relationships is presented, which allows combining the use of graph search algorithms with the subsequent visual processing of the obtained results based on visualization metaphors. Examples of visualization of situations that may characterize the incompleteness or redundancy of a set of cause-and-effect relationships between concepts are considered. It is shown that the effectiveness of cognitive model verification can be increased by increasing cognitive clarity of the visual image of the underlying FCM.

Areas for further research include:

- Development and research of other visualization metaphors useful in FCM verification. 
- Software implementation of the developed methodology in the form of a cognitive model verification subsystem as part of IGLA DSS [12], as well as its performance evaluation in the construction and study of fuzzy cognitive models of real applied problems.

The reported study was funded by RFBR, project number 19-07-00844.

\section{References}

1. Borisov, V.V., Kruglov, V.V., Fedulov, A.S.: Nechetkie modeli i seti [Fuzzy Models and Networks]. Goryachaya Liniya - Telekom, Moscow, Russia (2012). [in Russian]

2. Podvesovskii, A.G., Isaev, R.A.: Visualization Metaphors for Fuzzy Cognitive Maps. Scientific Visualization 10 (4), 13-29 (2018). doi: 10.26583/sv.10.4.02

3. Podvesovskii, A.G., Isaev, R.A.: Constructing Optimal Visualization Metaphor of Fuzzy Cognitive Maps on the Basis of Formalized Cognitive Clarity Criteria. Scientific Visualization 11 (4), 115-129 (2019). doi: 10.26583/sv.11.4.10

4. Zakharova, A.A., Shklyar, A.V.: Visualization Metaphors. Scientific Visualization 5 (2), 16-24 (2013).

5. Tiuriumin, V., Massel A.: Integration of Situation Semantic Models Based on Ontology System. In: Proceedings of 3rd Russian-Pacific Conference on Computer Technology and Applications (RPC). pp. 1-5. IEEE, Vladivostok, Russia (2018). doi: 10.1109/RPC.2018.8482232

6. Kulinich, A.A.: Verifikatsija kognitivnyh kart na osnove ob'jasnenija prognozov [Cognitive Maps Verification Based on Processes Explanation]. Upravlenie bol'shimi sistemami [Large-Scale Systems Control] 30.1, 453-469 (2010). [in Russian].

7. Podvesovskii, A.G., Isaev, R.A.: Identification of Structure and Parameters of Fuzzy Cognitive Models: Expert and Statistical Methods. International Journal of Open Information Technologies 7(6), 35-61 (2019).

8. Zakharova, A.A., Vekhter, E.V., Shklyar, A.V.: Using Visualization Tools to Search for Contradictions in Planning Results. Scientific Visualization 11 (4), 81-89 (2019). doi: 10.26583/sv.11.4.07

9. Cormen, Th.H., Leiserson, C.E.; Rivest, R.L.; Stein, C.: Introduction to Algorithms. 3rd, MIT Press (2009).

10. Abramova, N.A., Voronina, T.A., Portsev, R.Y. O metodah podderzhki postroeniya i verifikacii kognitivnyh kart $\mathrm{s}$ primeneniem idej kognitivnoj grafiki [Ideas of Cognitive Graphics to Support Verification of Cognitive Maps]. Upravlenie bol'shimi sistemami [Large-Scale Systems Control] 30.1, 411-430 (2010). [in Russian].

11. Lee, Y.-Y., Lin, C.-C., Yen, H.-C.: Mental Map Preserving Graph Drawing Using Simulated $\begin{array}{lllll}\text { Annealing. } & \text { Information } & \text { Sciences } & \text { 181(19), } & 4253-4272\end{array}$ doi: 10.1016/j.ins.2011.06.005

12. Zakharova, A.A., Podvesovskii, A.G., Isaev, R.A. Matematicheskoe i programmnoe obespechenie podderzhki kognitivnogo modelirovanija slabostrukturirovannyh organizacionno-tehnicheskih system [Mathematical and Software Support for Cognitive Modeling of Semi-structured Organizational and Technical Systems]. In: CPT2019 International conference Proceedings, pp. 131-141. Pub. NNGASU and SRCIPT, Nizhniy Novgorod (2019). [in Russian]. 